\title{
La prescriptora publicitaria de belleza en España y sus mecanismos de representación: Análisis a través de la revista Telva
}

The Advertising Prescriber of Beauty in Spain and its Mechanisms of Representation: Analysis done through Telva Magazine

Pilar Vicente Fernández Doctoranda EID - Universidad Rey Juan Carlos pilar.vicente@urjc.es

Dr. Isidoro Arroyo Almaraz

Profesor titular de universidad Universidad Rey Juan Carlos isidoro.arrroyo@urjc.es
Vicente Fernández, P. y Arroyo Almaraz, I. (2020) La prescriptora publicitaria de belleza en España y sus mecanismos de representación: Análisis a través de la revista Telva

Revista Internacional de Investigación en Comunicación aDResearch ESIC. № 22 Vol 22

Monográfico especial, marzo 2020 · Págs. 34 a 59

https://doi.org/10.7263/adresic-022-02 


\section{Clasificación JEL: M31, M37}

Palabras clave:

Prescriptora, comunicación, marketing, publicidad gráfica, Telva, mujer
Objetivo del estudio: La dimensión social y cultural del mensaje comercial atestigua la vinculación entre la publicidad y el contexto histórico en el que acontece, afectando al binomio mujer y comunicación desde diversas perspectivas; así, esta investigación tiene como propósito analizar la evolución de la imagen de la mujer en la publicidad atendiendo a su rol como prescriptora de mensajes comerciales relativos a marcas y productos de belleza, circunscribiéndose el trabajo a los últimos 56 años de la Historia de la Publicidad gráfica en España.

Diseño/metodología/enfoque: Se utiliza una metodología exploratoria y descriptiva mediante la técnica de análisis de contenido que se aplica a la totalidad de los anuncios gráficos del sector Belleza e Higiene insertados durante los meses de diciembre en Telva, cuya elección se sustenta por ser la revista femenina de alta gama con mayor antigüedad en el país de investigación. La muestra comprende desde el año 1963, fecha del primer ejemplar, hasta 2018, siendo objeto de estudio 1020 anuncios gráficos.

Resultados: Se evidencian diferencias en el tratamiento de la figura de la mujer como prescriptora publicitaria en función de la década analizada, siendo comunes no obstante un marcado carácter aspiracional y connotativo en la predicación así como un exiguo protagonismo del actante masculino como acompañante en la labor de prescripción. Se concluye la solidez de la impronta femenina en una comunicación de mujeres destinada a mujeres.

Limitaciones/implicaciones: El caso se restringe al medio gráfico revistas, pudiéndose plantear futuras investigaciones complementarias sobre análisis comparativos entre varios medios y distintos sectores de actividad empresarial.

Originalidad/contribución: Sobresalen la extensión y el alcance de la época de estudio cubierta, que permiten profundizar en los fundamentos de la prescripción publicitaria imperante en la actualidad y señalar las particularidades más destacadas de la prescripción de marcas y productos cosméticos.

\section{ABSTRACT}

\section{JEL Classification: M31, M37}

\section{Key words:}

Prescriber, communication, marketing, graphic advertising, Telva, woman
Purpose: The social and cultural dimension of the commercial message attests to the link between advertising and the historical context in which it takes place, affecting women and communication from various perspectives. Therefore, this research aims to analyze the evolution of the image of women in advertising according to their role as prescribers of commercial messages related to brands and beauty products in the last 56 years of the History of Graphic Advertising in Spain.

Design / Methodology / Approach: An exploratory and descriptive methodology is used via the technique of content analysis that applies to all the graphic advertisements of the Beauty and Hygiene sector inserted during the months of December in Telva, whose choice is based on the fact that it is the oldest high-end women's magazine in the country of research. The sample covers from the year 1963, date of the first issue, until 2018, having studied 1020 graphic advertisements.

Results: There were differences in the treatment of the figure of women as advertising prescriber according to the decade analyzed, being common, however, a marked aspirational and connotative character in preaching as well as a small role for the male participant as a companion in the work of prescribing. The solidity of the feminine imprint is concluded in a women's communication aimed at women.

Limitations / Implications: The case is restricted to the graphic medium journals, and it is possible to propose future complementary research on comparative analyses between various media and different sectors of business activity.

Originality / Contribution: The extent and scope of the time of study covered stand out, allowing one to delve into the fundamentals of the advertising prescription prevailing today as well as highlighting the most outstanding particularities of the prescription of brands and cosmetic products. 


\section{Introducción}

El término prescriptor en comunicación publicitaria resurge con fuerza en la actualidad motivado por la expansión y la consolidación de los prescriptores digitales sobrevenidos a raíz de las Tecnologías de la Información y la Comunicación. De este modo, expresiones como prescriptores 2.0 e influencers son habituales en la literatura científica del siglo XXI. Sin embargo, el prescriptor publicitario es un procedimiento considerablemente empleado por la comunicación de índole comercial durante toda su trayectoria, en su afán por satisfacer los intereses de marketing conectando con sus públicos gracias a su gran capacidad de recomendación de marcas y productos.

Así, se sugiere como pertinente revisar los precedentes y reflexionar acerca del desarrollo del recurso de los prescriptores publicitarios con el fin de vislumbrar el recorrido histórico y las características singulares del uso de esta figura a lo largo de la Historia de la Publicidad en nuestro país, focalizándose el estudio en la publicidad tradicional canalizada a través de los medios de comunicación de masas o medios convencionales. De entre ellos, el medio gráfico se erige como idóneo para sostener esta tipología de análisis, por su presencia notable en la segunda mitad del pasado siglo XX y por combinar texto e imagen dotando a la pieza publicitaria de una amplia variedad de códigos que redundan en una riqueza de matices y detalles distintivos en cada uno de los anuncios.

Desde el prisma de la comprensión del consumo bajo una visión integral de contexto social y cultural, entendiendo las relaciones que se establecen entre consumo, comunicación y cultura (Martín, 2013), se elige abordar la prescriptora publicitaria en España y sus mecanismos de representación por ostentar un papel preeminente en la publicidad debido a su labor de decisión, orientación y asesoramiento en diversas áreas de negocio. Se trata de una mujer convertida en consumidora propia, un paso más a la adquisición de mercancías para el núcleo familiar (Luque y Pérez, 2018). Esta investigación se centra en la publicidad de marcas y productos de cuidado personal ya que la categoría Belleza e Higiene, nomenclatura según la clasificación de InfoAdex, es una de las más prósperas y demandadas por parte de la mujer, así como un referente en cuanto a novedades y tendencias en la comunicación enfocada al target femenino. Los anuncios gráficos se ajustan a las inserciones publicitarias recogidas en los meses de diciembre en la veterana revista Telva, la más longeva de las revistas femeninas de alta gama en España.

En nuestro país, investigaciones previas vinculadas a la prescripción publicitaria cuestionan la imagen de la mujer y del hombre en comunicación comercial desde una perspectiva de género (Hidalgo-Marí, 2015; Martínez-Sanz y González, 2018; Rey, 2018; Vega, Barredo y Merchán, 2019), los contenidos y estereotipos en torno a un segmento de edad (Torres y García, 2015; Castelló, del Pino y Tur-Viñes, 2016; GonzálezAnleo, Cortés y Garcelán, 2018), el medio gráfico en sí (Almansa-Martínez y Gómez de TravesedoRojas, 2017; Benito, 2018; Treviños y Díaz-Soloaga, 2018; Viñuelas-López, 2019) e incluso debaten acerca de la cabecera objeto de la presente investigación (Roca, 2006).

No obstante lo anterior, y ante la constatación de una tradición de estudios que consideran esta temática, la originalidad y novedad de este proyecto radica en proporcionar un enfoque diacrónico y longitudinal de la figura de la prescriptora publicitaria en España a través de la citada revista madrileña, apostando por la extensión y 
el alcance del trabajo de campo de cara a una exhaustiva y rigurosa aproximación al objeto de análisis.

En base a todo lo preliminarmente expuesto, conviene subrayar el carácter exploratorio y descriptivo de esta investigación y recalcar que su principal objetivo consiste en estudiar la presencia de la prescriptora publicitaria de belleza a lo largo de los últimos 56 años de la Historia de la Publicidad gráfica en España, con la finalidad de perfilar los antecedentes de la vigente prescripción publicitaria femenina. Este propósito general se concreta en los siguientes objetivos específicos:

- Explorar los patrones compositivos y las tendencias de este tipo de discurso comunicativo década a década desde el punto de vista de la imagen de la mujer y cómo se articula mediante ella la predicación de marcas y productos cosméticos.

- Conocer la representación de la mujer prescriptora de belleza a través de sus características físicas y arquetipos.

- Examinar el peso y la función de los personajes masculinos como acompañantes de la figura femenina en los anuncios, así como su cometido en cuanto al anclaje y el significado del concepto publicitado.

- Entrever las inquietudes estéticas y de cuidado del físico de las españolas a través de las categorías de bienes dominantes en cada etapa histórica analizada.

La hipótesis de partida plantea que, a pesar de los cambios contextuales, el uso de la mujer como prescriptora publicitaria de belleza revela similares anhelos, motivaciones e intereses, los cuales configuran el sustento de la identificación entre prescriptora y destinataria de la comunicación comercial.

\section{Marco teórico}

\subsection{La prescripción publicitaria}

El verbo prescribir, que procede etimológicamente del latín praescriběre, significa «preceptuar, ordenar, determinar algo» (Real Academia Española, 2018). Más allá de sus frecuentes aplicaciones a nivel legal y médico, posee diversas acepciones que varían de acuerdo al entorno en que se encuadra.

Una primera revisión bibliográfica acerca del vocablo prescriptor en comunicación alude inequívocamente al de líder de opinión en la esfera de la opinión pública en política (Lazarsfeld, Berelson y Gaudet, 1944). Las cualidades de estos individuos, cuya personalidad cautivadora y carismática para persuadir trasciende el ámbito político, han sido estudiadas de manera interdisciplinar desde diferentes ópticas en aras de indagar en un mejor entendimiento del liderazgo de opinión como fenómeno.

El marketing y la comunicación publicitaria también han demostrado de forma temprana su interés por el papel que los líderes de opinión desempeñan en el marco de la actividad comercial en lo relativo a la consecución de objetivos económicos y de imagen (Chan y Misra, 1990). En ocasiones son celebridades o personalidades relevantes cuyos atributos se pretenden yuxtaponer a los de las marcas y los productos que sirven (Erdogan, 1999; Amos, Holmes y Strutton, 2008; Tzoumaka, Tsiotsou y Siomkos, 2016), partiendo de la investigación pionera de McCracken (1989) y su explicación del Modelo de Transferencia de Significados.

En España, este recurso se recoge inicialmente en obras que versan sobre Historia de la Publicidad (García y Fernández, 1990; Eguizábal, 1998; Castrillo y Oksman, 2007). Estas fuentes realzan la huella de este sujeto en la historia de nuestro país y apuntan cómo estos primeros pres- 
criptores publicitarios, actores y modelos anónimos mayoritariamente, adquirían un rol concreto (médico, tendero, deportista, ama de casa...) para influir a través de él en los comportamientos de los públicos, concienciando acerca de un hábito o incentivando la compra de un determinado producto o servicio. Investigaciones paralelas deliberan sobre la aproximación al concepto de prescriptor; así, es «aquel personaje que respalda el producto anunciado como experto, famoso o figurante» (Mondría, 2004, p. 235), matizando al respecto que «en el terreno del marketing, un prescriptor es alguien que, de forma individual, por su personalidad o por su pertenencia a una entidad, genera corrientes de influencia por sus opiniones, valoraciones y decisiones de compra» (Castelló y del Pino, 2015, p. 92).

Se reflexiona asimismo acerca de los requisitos que debe cumplir la prescripción para que la asociación entre prescriptor y marca / producto pueda conseguir un rendimiento provechoso. La coherencia es clave (Aguirre, 2010), sumándose la credibilidad, la confianza y la aspiracionalidad como factores concluyentes sobre los que trabajan la transferencia, la identificación y el influjo (Castelló y del Pino, 2015). Especial mención precisa el atributo de la credibilidad, que puede ser descrito en base a experiencia, honradez y atractivo físico (Ohanian, 1990), siendo este último fundamental en un primer momento de exposición del mensaje para captar la atención. Favorecen igualmente la fiabilidad y el halo de autoridad y competencia que emanan como emisores. Además, como señala Taveras, el prescriptor supone un activo para anunciantes y marcas (2014) y representa un arquetipo que canaliza la idea de placer que posibilitan marca y producto (2016).

Todo ello repercute en un importante valor añadido que afecta a la percepción de la variable precio de la estrategia de marketing, hasta el pun- to de estar el consumidor dispuesto a pagar más cuando una marca o un producto es avalado por un prescriptor con tales cualidades (Rumschisky, 2009).

\subsection{Las revistas femeninas de alta gama en España: el caso de Telva}

La prensa femenina, delimitada por su especialización temática «relacionada con la socialización diferencial de las mujeres» (Menéndez y Figueras, 2013, p. 31), goza de un largo recorrido en España. Tal y como establecen las mismas autoras, suele admitirse su surgimiento a finales del siglo XVIII con La Pensadora Gaditana, el primer periódico femenino, dirigido y redactado por Beatriz Cienfuegos. Sin embargo el afianzamiento de las revistas femeninas no se produce hasta mediados del siglo XIX con publicaciones sobre modas y labores esencialmente, cuya evolución convergerá durante las décadas de 1960 y 1980 en la concepción de la prensa femenina que se tiene en la actualidad, a lo que contribuye la llegada masiva de poderosos grupos editoriales extranjeros.

La revista femenina de alta gama es un tipo de prensa especializada dentro de las revistas de consumo y frente a las publicaciones de naturaleza profesional (Cabello, 1999). Siendo el esparcimiento un pilar elemental de su razón de ser, ejemplifica un sector de ingente envergadura en el mercado de las industrias culturales al caracterizarse por brindar contenidos variados de gran valor, un esmerado diseño gráfico y una destacable calidad fotográfica, circunstancias todas ellas que despiertan el interés de medianos y grandes anunciantes ávidos por destinar en estas revistas parte del presupuesto de marketing orientado a la compra de espacios publicitarios.

Esta clase de prensa de alta gama «está conformada por publicaciones periódicas centradas sobre todo en moda y belleza y va dirigida a la 
mujer, principal consumidora del mercado de revistas mensuales. Se trata de uno de los pocos productos de la cultura de masas escrito por, para y sobre mujeres, abarca temas definidos culturalmente como femeninos y propaga ideales de feminidad y belleza» (Torres, 2007, p. 213).

La cabecera madrileña Telva, fundada en 1963 y hoy parte del Grupo Unidad Editorial, constituye un soporte clave en la Historia de la Comunicación Social en nuestro país al ostentar más de 50 años ejerciendo influencia en la sociedad española. Englobada en el grupo de revistas femeninas cuyo eje de temáticas gira en torno al trinomio belleza - amor - hogar (Gallego, 1990), es la revista femenina más antigua de las que se publican actualmente en el panorama editorial español, aspecto que desemboca en poder ofrecer una perspectiva evolutiva, global e integradora del fenómeno investigado: la imagen de la mujer como prescriptora publicitaria en los anuncios de la categoría Belleza e Higiene que pueblan las páginas de su versión impresa.

El nacimiento de Telva en octubre de 1963, de la mano de SARPE y bajo la dirección de una pionera Pilar Salcedo, conlleva «una ruptura con el modelo de prensa femenina en España» (Menéndez y Figueras, 2013, p. 40) en un contexto de renovación y desarrollo económico. Al precio de 6 pesetas, suma 56 páginas y su tirada es de 40.000 ejemplares. De carácter conservador como seña de identidad y con una periodicidad quincenal, se presenta, como expone la carta de bienvenida de su directora, con el compromiso de «cumplir el orden riguroso de toda prensa honrada: informar, formar y distraer» $\mathrm{y}$ con la voluntad de utilidad como criterio distintivo, contemplando los ingresos publicitarios como vía de financiación.

«Telva se ha convertido en la gran revista femenina española por excelencia» (Ganzabal,
2006, p. 417), capaz de ir adaptándose a los cambios a lo largo de sus prolíficas décadas de existencia. Como revista autóctona de vital relevancia dentro de la prensa femenina de nuestro país, desde los inicios de su producción colabora en la propagación social de marcas y productos de moda y estética, creando estructuras de significado que responden a necesidades y deseos enmarcados en entornos físicos y culturales cambiantes (Martín, 2013).

\subsection{La comunicación de belleza}

El arranque de Telva coincide con transformaciones en la mentalidad de los españoles: «A partir de 1960 los cuidados del cuerpo, superando ancestrales complejos y reservas de orden moral o religioso, dieron lugar al consumo de productos como jabones, perfumes, masajes, desodorantes, maquillajes, etc.» (Eguizábal, 1998, p. 485); la alimentación deja de ser el problema esencial del español medio para convivir con inquietudes relacionadas con las comodidades hogareñas y el bienestar físico.

Desde entonces, la belleza es, tal y como se ha advertido y puntualiza Torres (2007), una de las grandes motivaciones de la lectora de una revista de alta gama, encumbrándose como el segundo tema de mayor presencia cuantitativa en estas publicaciones, únicamente por detrás de la moda. Según la misma autora, «la preocupación por la esbeltez, la juventud y la perfección física es acusada; tal apariencia se presenta como icono cultural» (p. 217). Idea con la que concuerdan Magaña (2014) al hablar de una sociedad gobernada, entre otros factores, por la veneración a la belleza y al aspecto físico; y Treviños y Díaz-Soloaga (2018), quienes sentencian al respecto que la comunicación perpetúa de forma estereotípica un modelo de belleza canónico, en concreto el de una mujer «joven, predominantemente blanca, 
delgada y rodeada por un aura de poder y estatus» (Soley-Beltrán, 2012, p. 123). Este patrón de estética marcadamente occidental se expande a múltiples ámbitos culturales (Fowler y Carlson, 2015), implementándose un canon de belleza hegemónico, restrictivo y normativo así como unos prototipos corporales que confluyen en una visión idealizada de la mujer (Del Rosso, 2016).

De ahí que comunicación y publicidad sobre el sector Belleza e Higiene sean más que notorias en las revistas femeninas de alta gama. En ellas las mujeres localizan sugerencias y consejos mediante los cuales y de manera cómplice estas publicaciones consiguen crear una atmósfera íntima a modo de punto de encuentro en el que hallar respuestas para supuestamente reforzar su seguridad y autoestima, combinándose y solapándose objetivos y contenidos de información, persuasión y entretenimiento.

Así, sobre el retrato de la belleza en la prensa femenina de alta gama, editores de revistas y anunciantes adoptan una «tecnología del encanto» como vía para ejercer control sobre sus lectoras, dotando al lenguaje de las revistas y de la publicidad de poder «mágico» (Moeran, 2010, p. 492). Todo ello inmerso en recursos retóricos a los que la creatividad publicitaria apela para su fin último de sugestión y persuasión (Arroyo, 2005).

En este ambiente, en el que las marcas son conocedoras de la magnitud de la confianza, la seducción y la empatía que se precisan en el marketing de productos de cuidado personal, despunta el empleo de la prescriptora publicitaria como escaparate en el que la mujer pueda proyectarse y como fuente de anhelos en la que inspirarse.

De este modo, en el sector Belleza e Higiene «la publicidad confiere a la mercancía un valor simbólico que la lectora desea, como el atractivo y la distinción, con el que la consumidora pasa a auto-definirse y a identificarse, conforme la con- sume» (Torres, 2007, p. 221). Los mensajes publicitarios sobre marcas y productos relativos a la industria de la belleza, cuyo discurso es eminentemente de corte hedonista, constatan cómo el campo de la cosmética trasciende los límites del bien en sí para alzarse en un espejo del estilo de vida de la usuaria y en un reflejo de su identidad, así como en un canal en el que buscar felicidad y autorrealización (Aranda, 2018).

\section{Metodología}

La oportunidad de esta investigación es un dilema de condición social y cultural en el territorio de la comunicación publicitaria. La estrategia metodológica considerada más adecuada para tal meta es el análisis de contenido, «técnica destinada a formular, a partir de ciertos datos, inferencias reproducibles y válidas que puedan aplicarse a un contexto» (Krippendorff, 1989, p. 403).

\subsection{Periodo de análisis y muestra}

La observación y el análisis documental propios del análisis de contenido (López-Aranguren, 2010) se efectúan en los fondos documentales localizados en distintas unidades de información: Biblioteca Nacional, Hemeroteca Municipal de Madrid y sede del Grupo Unidad Editorial, en este último caso gracias a la persona responsable de Archivo Gráfico y Documentación de Telva.

Con el objetivo de ahondar en la complejidad y profusión del fenómeno investigado se opta por potenciar su carácter longitudinal y contemplar como periodo de análisis desde el año 1963, fecha del primer número de la revista Telva, hasta 2018, por ser el más cercano al cierre de la investigación. Siendo el universo la totalidad de los ejemplares de la revista en soporte papel publicados desde su nacimiento en octubre de 1963, la muestra se acota en base al criterio de una elevada inversión publicitaria por parte de los anunciantes, lo que 
habitualmente acaece durante el mes de diciembre (Aguilera, 2015) por su proximidad a las fiestas navideñas y la conexión de esta época con el consumo exacerbado y los regalos. De esta forma se procede a la búsqueda y al registro de todos los números de diciembre de la revista Telva desde 1963 hasta 2018. Al ser quincenal la periodicidad de la publicación desde 1963 hasta finales de 1989, momento en el que pasa a ser mensual, se consultan 82 ejemplares en total.
En estos 82 números, las unidades de análisis seleccionadas se corresponden con los anuncios publicitarios impresos que cuentan con el recurso de una prescriptora femenina o parte de su figura, de cualquier formato y cuya marca y producto se integran en el sector de Belleza e Higiene. Tras la cuantificación en el periodo señalado, se registran 1165 unidades de análisis. Excluyendo repeticiones, el corpus de estudio final se compone de 1020 anuncios.

\section{Tabla 1. Registro y cuantificación de las unidades de análisis}

\begin{tabular}{|c|c|c|c|c|c|c|c|c|c|c|c|c|c|c|c|c|c|c|c|}
\hline \multicolumn{20}{|c|}{ Anuncios «Belleza e Higiene» - Revista Telva } \\
\hline \multicolumn{3}{|c|}{ 1a. década } & \multicolumn{3}{|c|}{ 2a. década } & \multicolumn{3}{|c|}{ 3a. década } & \multicolumn{3}{|c|}{ 4a. década } & \multicolumn{3}{|c|}{ 5a. década } & \multicolumn{3}{|c|}{ 6a. década } & \multirow{2}{*}{$\begin{array}{l}\text { Total } \\
1165\end{array}$} & \multirow{2}{*}{$\begin{array}{c}\text { Sin } \\
\text { repet }\end{array}$} \\
\hline Año & $\begin{array}{c}\text { Anun- } \\
\text { cios }\end{array}$ & - Repet & Año & $\begin{array}{c}\text { Anun- } \\
\text { cios }\end{array}$ & - Repet & Año & $\begin{array}{c}\text { Anun- } \\
\text { cios }\end{array}$ & Repet & Año & $\mid \begin{array}{c}\text { Anun- } \\
\text { cios }\end{array}$ & Repet & Año & $\mid \begin{array}{c}\text { Anun- } \\
\text { cios }\end{array}$ & Repet & t Año & $\begin{array}{c}\text { Anun- } \\
\text { cios }\end{array}$ & Repet & & \\
\hline 1963 & 0 & 0 & 1973 & 19 & 2 & 1983 & 15 & 0 & 1993 & 19 & 4 & 2003 & 39 & 3 & 2013 & 24 & 3 & & \\
\hline 1964 & 10 & 0 & 1974 & 17 & 1 & 1984 & 18 & 2 & 1994 & 24 & 5 & 2004 & 34 & 1 & 2014 & 22 & 4 & & \\
\hline 1965 & 12 & 0 & 1975 & 15 & 5 & 1985 & 15 & 2 & 1995 & 24 & 1 & 2005 & 21 & 6 & 2015 & 25 & 3 & & \\
\hline 1966 & 12 & 2 & 1976 & 13 & 1 & 1986 & 18 & 2 & 1996 & 24 & 7 & 2006 & 32 & 1 & 2016 & 22 & 3 & & \\
\hline 1967 & 16 & 0 & 1977 & 12 & 1 & 1987 & 28 & 4 & 1997 & 27 & 3 & 2007 & 34 & 7 & 2017 & 26 & 3 & & \\
\hline 1968 & 20 & 2 & 1978 & 10 & 1 & 1988 & 22 & 2 & 1998 & 33 & 5 & 2008 & 23 & 3 & 2018 & 18 & 2 & & \\
\hline 1969 & 23 & 4 & 1979 & 7 & 1 & 1989 & 20 & 4 & 1999 & 35 & 6 & 2009 & 28 & 2 & & & & & \\
\hline 1970 & 16 & 2 & 1980 & 15 & 0 & 1990 & 16 & 5 & 2000 & 27 & 8 & 2010 & 27 & 1 & & & & & \\
\hline 1971 & 20 & 2 & 1981 & 6 & 1 & 1991 & 19 & 1 & 2001 & 33 & 8 & 2011 & 28 & 1 & & & & & \\
\hline 1972 & 13 & 2 & 1982 & 6 & 0 & 1992 & 21 & 2 & 2002 & 34 & 3 & 2012 & 28 & 1 & & & & & \\
\hline & 142 & 14 & & 120 & 13 & & 192 & 24 & & 280 & 50 & & 294 & 26 & & 137 & 18 & & \\
\hline \multicolumn{3}{|c|}{$\%$ Repet } & \multicolumn{3}{|c|}{$10,83 \%$} & \multicolumn{3}{|c|}{$12,50 \%$} & \multicolumn{3}{|c|}{$17,85 \%$} & \multicolumn{3}{|c|}{$8,84 \%$} & \multicolumn{3}{|c|}{$13,13 \%$} & & \\
\hline \multicolumn{3}{|c|}{ Sin Repet } & \multicolumn{3}{|c|}{ Sin Repet } & \multicolumn{3}{|c|}{ Sin Repet } & \multicolumn{3}{|c|}{ Sin Repet } & \multicolumn{3}{|c|}{ Sin Repet } & \multicolumn{3}{|c|}{ Sin Repet } & & \\
\hline \multicolumn{3}{|c|}{128} & \multicolumn{3}{|c|}{107} & \multicolumn{3}{|c|}{168} & \multicolumn{3}{|c|}{230} & \multicolumn{3}{|c|}{268} & \multicolumn{3}{|c|}{119} & & \\
\hline
\end{tabular}




\subsection{Variables y categorización}

Se confecciona una plantilla de análisis que persigue constituir un modelo de estudio práctico y eficiente para sistematizar el registro de las variables objeto de la investigación y obtener inferencias cuantitativas, cumpliéndose los imperativos por los que abogan Wimmer y Dominick (1996): sistemático, objetivo y cuantitativo.

Este instrumento se realiza teniendo en cuenta los siguientes sistemas de variables o categorías de análisis globales:

- Datos de identificación: año, marca, producto, subcategoría/s, formato publicitario, objetivo de comunicación, camino/s creativo/s, otras acciones de comunicación.

- Código escritural: titular, cuerpo de texto, frase de cierre, eslogan, contenido de la predicación (características de marca/producto, reason why de la promesa, beneficios y valores derivados), referencia a país/ciudad de origen, argumentación, fórmula de tratamiento, idioma de texto, tono de la comunicación.

- Código tipográfico: estilo y color, signos (interrogación, exclamación), puntos suspensivos, comillas, efectos.

- Código cromático: pigmento, clasificación, estética predominante.

- Código fotográfico: formato, encuadre de la figura humana, tipo de plano, ángulo de proyección, iluminación, fondo, localización.

- Código sociocultural: número de actantes, género e interrelación; en caso de personaje masculino: notoriedad, arquetipo y rol; sobre la figura femenina prescriptora: notoriedad, predicación, arquetipo, rol, raza, segmento de edad, complexión, cabello, vestuario, atrezzo, otros elementos.

- Código gestual: parte visible de la mujer, pose de la cabeza, mirada, labios, expresiones facial y corporal, relación con el producto.
- Código morfológico: composición, construcción de la imagen, integración de marca y producto, packshot y su ubicación.

\section{Resultados}

Considerando los objetivos perseguidos y la metodología utilizada, se presentan los principales hallazgos de la investigación organizados por décadas debido a la amplitud y al volumen de la muestra.

\subsection{De 1963 a 1972:}

En esta década iniciática, la subcategoría «Colonias y perfumes» representa el 32,37 \% del total de los anuncios de Belleza e Higiene, siendo mayoritarios los femeninos (30,21 \%). La marca española Myrurgia, con sus emblemáticas Joya o Nueva Maja, es la que más invierte en publicidad. Le sigue «Maquillaje» $(26,61 \%)$, destacando los productos para labios, ojos y uñas (15,82\%). A continuación, «Tratamientos de belleza faciales» $(24,46 \%)$. Completan el reparto «Cuidados del cuerpo» (5,03\%), «Higiene femenina» (4,31\%) $\mathrm{y}$ «Productos para el cabello» $(4,31 \%)$.

La figura de la prescriptora publicitaria de estos productos se enmarca en una publicidad gráfica cuyo formato primordial es la página (67,18 \%), seguida de la media página vertical (21,87\%). A través de ellos desempeña su rol prescriptor reiterando la novedad del producto en un $32 \%$ de las ocasiones. El camino creativo de la exhibición de beneficios y valores de la promesa es el protagonista, de ahí la importancia de la representación de la imagen femenina. Otros son el testimonial y el problema - solución, combinados con referencias al paso a paso y los trozos de vida. Recursos retóricos como la analogía, los juegos de palabras y la personificación del producto en la prescriptora asisten a la labor de recomendación.

Los elementos escriturales gozan de gran presencia, sobre todo cuerpo de texto (72\%) y titular 
(67\%). Mediante ellos la predicación sobre marca y producto enfatiza los efectos de la promesa publicitada (32\%), y en un $25 \%$ de los casos profundiza en tales consecuencias junto a las propiedades de marca/producto y al reason why que las sustenta. «Belleza», «juventud», «perfección» y «seducción» son los atributos capitales derivados del empleo del bien, concebido como solución o milagro y acentuado por adjetivos como «sorprendente», «maravilloso» o «sensacional». La argumentación que aúna matices racionales y emocionales es la habitual, con un estilo explicativo y a la vez sugerente y distinguido. Se trata de una publicidad descriptiva, de los porqués y con carácter periodístico de noticia, tratamiento predominante de usted e idioma español para facilitar la asimilación del contenido. Tipográficamente, mayúsculas y negrita recalcan algunas palabras, además de puntos suspensivos (32\%) y comillas (29\%).

La mayoría son anuncios en blanco y negro $(58,60 \%)$ con una fotografía (76 \%), si bien se usa la ilustración (10 \%) de manera simbólica para actitudes y posturas más atrevidas y desenfadadas. La composición individual, con una mujer como único actante, es propia del 80,50 \% de los anuncios. Desde un primer plano (30,45 \%) o un plano medio $(24,71 \%)$, una mujer anónima de raza blanca, adulta, complexión delgada, principalmente cabello rubio y vestuario y atrezzo ostentosos (maquillaje, joyas, adornos, brillos...) ejerce en solitario la prescripción de marca y producto, con un fondo liso o difuminado (80 \%) que busca centrar la atención en el rostro. Suele mantener la cabeza erguida y ladeada, mirando al espectador con una ligera sonrisa y sin interactuar con el producto (75 \%). Los arquetipos dominantes son «Preocupada por su belleza y el paso del tiempo» $(42,27 \%)$, «Elegante y sofisticada» $(20,32 \%)$ y «Mujer deseo» $(17,88 \%)$, siendo apenas significativo el de «Esposa, madre y ama de casa» $(4,06 \%)$.

Únicamente el 19,50 \% de los anuncios cuenta con más de un actante y solo en 16 se aprecia

\section{Tabla 2. Estadística descriptiva 1963-1972}

\begin{tabular}{|c|c|c|}
\hline \multicolumn{3}{|c|}{ Anuncios categoría «Belleza e Higiene» } \\
\hline Subcategorías & & $\%$ \\
\hline & Colonias y perfumes & $32,37 \%$ \\
\hline & Femeninos & $30,21 \%$ \\
\hline & Unisex & $1,45 \%$ \\
\hline & Familiares & $0,71 \%$ \\
\hline & Maquillaje & $26,61 \%$ \\
\hline & Tratamientos de belleza faciales & $24,46 \%$ \\
\hline & Cuidados del cuerpo & $5,03 \%$ \\
\hline & Higiene femenina & $4,31 \%$ \\
\hline & Productos cabello & $4,31 \%$ \\
\hline & Otros & $2,91 \%$ \\
\hline
\end{tabular}




\section{Tabla 2. Estadística descriptiva 1963-1972 (continuación)}

Formatos publicitarios mayoritarios

\begin{tabular}{|l}
\hline \\
\hline Lanzamiento marca / producto \\
\hline Código escritural \\
\hline
\end{tabular}

Página
$1 / 2$ pág. vertical

$67,18 \%$

Concepto «novedads

Concepto «novedad»

$21,87 \%$

-

\begin{tabular}{|l}
\hline Principales elementos \\
\hline Contenido predicación \\
\hline Código tipográfico
\end{tabular}

\begin{tabular}{l} 
Titular \\
\hline Cuerpo de texto \\
\hline
\end{tabular}

$32,00 \%$

\section{Eslogan}

Características + RW + Efectos

$67,00 \%$

Únicamente efectos de la promesa

$72,00 \%$

$33,00 \%$

$25,00 \%$

$32,00 \%$

\begin{tabular}{l} 
Recursos destacados \\
\hline Código cromático
\end{tabular}

\begin{tabular}{|l|l|}
\hline Puntos suspensivos & $32,00 \%$ \\
\hline Comillas & $29,00 \%$
\end{tabular}

Código fotográfico

$\mathrm{B} / \mathrm{N}$

$58,60 \%$

Color

$41,40 \%$

Principales planos

Primer plano

$30,45 \%$

Plano medio

$24,71 \%$

Relación actantes $\mathrm{H}$ y M

Arquetipos femeninos dominantes

Relación de pareja

$12,50 \%$

Relación de pareja implícita

$4,68 \%$

Preocupada por su belleza y el paso del tiempo

$42,27 \%$

Elegante y sofisticada

$20,32 \%$

Mujer deseo

$17,88 \%$

Esposa, madre y ama de casa

\section{Código gestual}

Contacto físico con el producto

Código morfológico

Composición individual
Composición de pareja o grupal


relación de pareja. Además, cabe añadir 6 piezas con alusiones a una vinculación sentimental implícita a través del texto pero sin manifestación física del hombre en la imagen. Por tanto, la pareja se representa en 22 anuncios (17,18\%). El actante masculino es siempre anónimo, genérico (sin rostro o cortado), con traje oscuro, secundario y encarnando el rol «Hombre-hombre» como objeto de seducción (Tabla 2).

\subsection{De 1973 a 1982:}

Continúa el liderazgo de «Colonias y perfumes» (37,09\%), a causa de la supremacía de los femeninos y del ligero avance del territorio unisex. Junto a las nacionales Myrurgia o Puig se adhieren enseñas francesas de renombre como Nina Ricci o Christian Dior. «Maquillaje» (16,12 \%) pierde fuerza a favor de «Tratamientos de belleza faciales» (28,22 \%), y es que el esmero en el cuidado del rostro evidencia la reivindicación de la belleza natural como una de las aspiraciones cruciales de la década. Aumenta la inversión publicitaria en «Productos para el cabello» $(7,25 \%)$ y se mantiene para «Cuidados del cuerpo»e «Higiene femenina».

Permanece la página como formato publicitario por excelencia $(76,63 \%)$, decae la utilización de la media página vertical $(10,28 \%)$ y la doble página va ganando impulso $(5,60 \%)$. Fruto de una mayor apertura hacia el exterior, el número de lanzamientos es considerable ( $38,31 \%$ ). «Ahora» $\mathrm{u}$ «hoy» son adverbios para informar de novedades, y desde esta necesidad de dar a conocer las mercancías, el cuerpo de texto $(69,15 \%)$ y el eslogan $(54,20 \%)$ son elementos escriturales de apreciable trascendencia en la predicación, observándose la apuesta por el eslogan de cara a la construcción del posicionamiento de marca y producto. Se asiste a una publicidad explicativa en la que la prescriptora insiste en la predicación del reason why (37,38 \%), que posibilita una promesa de «simplicidad» y «pureza» gracias a fórmulas e ingredientes procedentes de la naturaleza que ayudan a conseguir una piel «hidratada» $y$ «luminosa». Una vez más la demostración se consolida como el camino creativo preferente, seguido del testimonial. Ocasiones de uso, interrogaciones retóricas y redundancias contribuyen a una predicación informativa, aspiracional y cómplice en lengua española, sin fórmula de tratamiento y con diversidad en el estilo y color de los tipos coincidiendo con el incremento de las piezas en color $(76,63 \%)$. Blanco o negro suelen contrastar con el dorado y con tonos de la naturaleza (ocre, azul, verde...).

La imagen de la mujer se materializa esencialmente en una única fotografía $(84,11$ \%) de composición individual $(78,50 \%)$. El primer plano $(27,10 \%)$ o el plano medio (23,36 \%) muestran a una mujer con las mismas características físicas y expresiones que en la década anterior, a pesar de que vestuario y atrezzo abogan más por la sencillez, la ausencia de artificios y un estilo romántico. Una localización visible y exterior (campo, mar...) sugiere evocación y ensoñación. El arquetipo «Preocupada por su belleza y el paso del tiempo» $(40,05 \%)$ convive con los de «Elegante y sofisticada» $(10,28 \%)$, «Sensual y soñadora» $(15,62 \%)$, con actitud más reflexiva y propio de la época, y «Mujer deseo» $(13,08 \%)$, seductora y enamorada, despuntando el rol tradicional como «Esposa, madre y ama de casa» hasta un $6,54 \%$.

Como novedad, el actante masculino va adquiriendo la condición de complementariedad como objeto de seducción y seductor a la vez, aunque tanto su presencia (13 anuncios) como la asunción de una relación de pareja (9,34 \%) decaen respecto al periodo previo. 


\section{Tabla 3. Estadística descriptiva 1973-1982}

\begin{tabular}{|c|c|c|}
\hline \multicolumn{3}{|c|}{ Anuncios categoría «Belleza e Higiene» } \\
\hline Subcategorías & & $\%$ \\
\hline & Colonias y perfumes & $37,09 \%$ \\
\hline & Femeninos & $31,45 \%$ \\
\hline & Unisex & $4,83 \%$ \\
\hline & Masculinos & $0,81 \%$ \\
\hline & Maquillaje & $16,12 \%$ \\
\hline & Tratamientos de belleza faciales & $28,22 \%$ \\
\hline & Cuidados del cuerpo & $4,03 \%$ \\
\hline & Higiene femenina & $5,64 \%$ \\
\hline & Productos cabello & $7,25 \%$ \\
\hline & Otros & $1,65 \%$ \\
\hline \multicolumn{3}{|c|}{ Formatos publicitarios mayoritarios } \\
\hline & Página & $76,63 \%$ \\
\hline & $1 / 2$ pág. vertical & $10,28 \%$ \\
\hline & Doble página & $5,60 \%$ \\
\hline Lanzamiento marca / producto & Concepto «novedad» & $38,31 \%$ \\
\hline \multicolumn{3}{|l|}{ Código escritural } \\
\hline \multirow{3}{*}{ Principales elementos } & Titular & $56,07 \%$ \\
\hline & Cuerpo de texto & $69,15 \%$ \\
\hline & Eslogan & $54,20 \%$ \\
\hline Contenido predicación & Características + RW + Efectos & $37,38 \%$ \\
\hline \multicolumn{3}{|l|}{ Código tipográfico } \\
\hline \multirow{2}{*}{ Recursos destacados } & Puntos suspensivos & $25,23 \%$ \\
\hline & Comillas & $27,10 \%$ \\
\hline \multicolumn{3}{|l|}{ Código cromático } \\
\hline & $\mathrm{B} / \mathrm{N}$ & $23,37 \%$ \\
\hline & Color & $76,63 \%$ \\
\hline \multicolumn{3}{|l|}{ Código fotográfico } \\
\hline \multirow{2}{*}{ Principales planos } & Primer plano & $27,10 \%$ \\
\hline & Plano medio & $23,36 \%$ \\
\hline
\end{tabular}




\section{Tabla 3. Estadística descriptiva 1973-1982 (continuación)}

\begin{tabular}{|c|c|c|}
\hline \multicolumn{3}{|l|}{ Código sociocultural } \\
\hline Relación actantes $\mathrm{Hy} \mathrm{M}$ & Relación de pareja & $9,34 \%$ \\
\hline \multirow{5}{*}{ Arquetipos femeninos dominantes } & Preocupada por su belleza y el paso del tiempo & $40,05 \%$ \\
\hline & Elegante y sofisticada & $10,28 \%$ \\
\hline & Sensual y soñadora & $15,62 \%$ \\
\hline & Mujer deseo & $13,08 \%$ \\
\hline & Esposa, madre y ama de casa & $6,54 \%$ \\
\hline \multicolumn{3}{|l|}{ Código gestual } \\
\hline & Contacto físico con el producto & $20,56 \%$ \\
\hline \multicolumn{3}{|l|}{ Código morfológico } \\
\hline & Composición individual & $78,50 \%$ \\
\hline & Composición de pareja o grupal & $21,50 \%$ \\
\hline
\end{tabular}

\subsection{De 1983 a 1992:}

Este lapso de tiempo supone la consagración de la subcategoría «Colonias y perfumes femeninos» $(54,33 \%)$, que junto a «Tratamientos de belleza faciales» $(28,32 \%)$ abanderan una comunicación publicitaria donde la figura prescriptora defiende conceptos innovadores como «estilo» o «independencia» de la mano de marcas francesas principalmente, tales como Yves Saint Laurent, Cacharel o Vichy, entre otras. «Maquillaje» cae hasta el 8,09 \%, desaparece la clásica subcategoría publicitaria de «Higiene femenina» y se atisba una tímida introducción de referencias sobre «Estética corporal» (4,62\%), particularmente peluquerías e institutos de estética.

La prescriptora publicitaria alza su voz a través de los formatos página (63,09 \%) y doble página $(28,57 \%)$, que se erigen como los más solicitados por los anunciantes del sector Belleza e Higiene. Disminuye el peso de los lanzamientos de productos $(29,16 \%)$ y de los componentes escritu- rales (43,45 \% cuerpo de texto y 36,30 \% eslogan), incorporando no obstante la prescriptora al discurso publicitario la propiedad «antiarrugas» como distintiva del 46,80 \% de las cremas hidratantes y nutritivas de la década. La explicación del reason why de la promesa sigue siendo importante, ilustrándose en ocasiones desde los ingredientes que marca y producto no poseen, en una predicación más elaborada y compleja para una receptora cada vez más habituada al léxico típico del cuidado personal.

Así, la evolución lógica de la publicidad del sector y de los conocimientos de su target al respecto propicia cada vez más argumentaciones de tintes emocionales. De este modo, expresiones como «magia», «misterio»y «atracción» envuelven la figura de la prescriptora publicitaria, en anuncios donde la explosión de color (87,50 \%) y la escasez de efectos tipográficos resaltan una estética de sofisticación y lujo de marcado carácter urbanita y con apelación al mundo de los sentidos. 
A través de una única foto $(89,28 \%)$ y primer plano $(40,47 \%)$ o plano medio (32,14\%), la mujer abandona el rol de «Esposa, madre y ama de casa» y afianza los arquetipos de «Preocupada por su belleza y el paso del tiempo» (34,52\%), «Elegante y sofisticada» $(32,73 \%)$ y «Sensual y soñadora» $(17,85 \%)$. «Mujer deseo» $(14,88 \%)$ comienza a apoderarse de rasgos de «Mujer fatal», más provocativa e hipnótica. Con cabello largo y suelto y expresión de seguridad y confianza, se presenta con los labios cerrados y mirada fuera de cámara. Grandes joyas, lentejuelas y pieles completan su vestuario y atrezzo en las composiciones, ascendiendo las individuales hasta regis- trar una fuerza del 83,33 \%. Cuando hay más de un actante (16,67\%), la composición protagonizada por féminas (11,30 \%) con relación de igualdad y complicidad supera a la que muestra un nexo de pareja entre hombre y mujer (5,37 \%), evidenciándose un contacto físico cada vez más acusado entre los actantes y no tanto con el producto objeto de la publicidad (solo en 18,45 \% de las piezas).

Por vez primera la prescripción se ejerce testimonialmente desde la condición de personaje notorio en un 6,54 \% de los anuncios, gracias a reconocidas modelos de prestigio y actrices que sintetizan la esencia de la promesa publicitada.

\section{Tabla 4. Estadística descriptiva 1983-1992}

\begin{tabular}{|c|c|c|}
\hline \multicolumn{3}{|c|}{ Anuncios categoría «Belleza e Higiene» } \\
\hline Subcategorias & & $\%$ \\
\hline & Colonias y perfumes & $54,90 \%$ \\
\hline & Femeninos & $54,33 \%$ \\
\hline & Unisex & $0,57 \%$ \\
\hline & Maquillaje & $8,09 \%$ \\
\hline & Tratamientos de belleza faciales & $28,32 \%$ \\
\hline & Cuidados del cuerpo & $1,73 \%$ \\
\hline & Productos cabello & $1,73 \%$ \\
\hline & Estética corporal & $4,62 \%$ \\
\hline & Otros & $0,61 \%$ \\
\hline \multicolumn{3}{|l|}{ Formatos publicitarios mayoritarios } \\
\hline & Página & $63,09 \%$ \\
\hline & Doble página & $28,57 \%$ \\
\hline Lanzamiento marca / producto & Concepto «novedad» & $29,16 \%$ \\
\hline \multicolumn{3}{|l|}{ Código escritural } \\
\hline \multirow{3}{*}{ Principales elementos } & Titular & $29,76 \%$ \\
\hline & Cuerpo de texto & $43,45 \%$ \\
\hline & Eslogan & $36,30 \%$ \\
\hline Contenido predicación & Características + RW + Efectos & $30,35 \%$ \\
\hline
\end{tabular}




\section{Tabla 4. Estadística descriptiva 1983-1992 (continuación)}

\begin{tabular}{|c|c|c|}
\hline \multicolumn{3}{|l|}{ Código tipográfico } \\
\hline \multirow{2}{*}{ Recursos destacados } & Mayúsculas & $14,88 \%$ \\
\hline & Comillas & $9,52 \%$ \\
\hline \multicolumn{3}{|l|}{ Código cromático } \\
\hline & $\mathrm{B} / \mathrm{N}$ & $12,50 \%$ \\
\hline & Color & $87,50 \%$ \\
\hline \multicolumn{3}{|l|}{ Código fotográfico } \\
\hline \multirow{2}{*}{ Principales planos } & Primer plano & $40,47 \%$ \\
\hline & Plano medio & $32,14 \%$ \\
\hline \multicolumn{3}{|l|}{ Código sociocultural } \\
\hline Prescriptora celebrity & Actrices, modelos de prestigio & $6,54 \%$ \\
\hline Relación actantes H y M & Relación de pareja & $5,37 \%$ \\
\hline \multirow{4}{*}{ Arquetipos femeninos dominantes } & Preocupada por su belleza y el paso del tiempo & $34,52 \%$ \\
\hline & Elegante y sofisticada & $32,73 \%$ \\
\hline & Sensual y soñadora & $17,85 \%$ \\
\hline & Mujer deseo / Mujer fatal & $14,88 \%$ \\
\hline \multicolumn{3}{|l|}{ Código gestual } \\
\hline & Contacto físico con el producto & $18,45 \%$ \\
\hline \multicolumn{3}{|l|}{ Código morfológico } \\
\hline & Composición individual & $83,33 \%$ \\
\hline & Composición de pareja o grupal & $16,67 \%$ \\
\hline
\end{tabular}

\subsection{De 1993 a 2002:}

En esta etapa, de notable inversión publicitaria, siguen aumentando los anuncios sobre «Colonias y perfumes femeninos» $(62,65 \%)$, aunque el porcentaje de la subcategoría «Tratamientos de belleza faciales» (17\%) se reduce al tiempo que «Maquillaje» (12,86 \%) adquiere un dato significativo, prestando especial atención a los productos para maquillar ojos y labios. Además, se recorta la cifra relativa a «Estética corporal» $(2,48 \%)$, y otros sectores como «Cuidados del cuerpo»y
«Productos para el cabello» apenas son relevantes. Marcas estadounidenses como Calvin Klein o Tommy Hilfiger empiezan a ocupar su espacio en un contexto de acentuado espíritu francés.

Página $(79,56 \%)$ y doble página $(20,44 \%)$ son los únicos formatos publicitarios localizados, percibiéndose casos de encartes y sampling como ejemplos de despliegue y progresión de tales formatos. Las novedades de marcas y productos $(27,82 \%)$, similares al periodo precedente, constatan la realidad de un mercado asentado y ma- 
duro, donde la simple identificación de marca y producto es suficiente en un 28,26\% de las piezas, prescindiendo de otros componentes escriturales como cuerpo de texto y eslogan, cuya funcionalidad merma de forma clara. La tendencia consiste en recurrir a una breve acotación sobre el objeto publicitado (44,34\%), subrayando la prescriptora la «experiencia» de la marca y haciendo hincapié en el «futuro»y la «prevención» como inquietudes imperantes.

La promesa publicitaria se focaliza en torno a argumentos emocionales asociados al mundo de las sensaciones («pasión», «locura», «atrevimiento», «evasión»...), siendo la «sensualidad»y la «feminidad» los ideales de esta década. Entronca con el aumento del testimonial como camino creativo: en 17,82 \% de los anuncios es una prescriptora famosa (diseñadora, actriz o súper modelo) quien encarna tales principios, reforzados por una tipografía de estilo manuscrito, más informal, y por matices cromáticos que connotan calidez (tono piel, naranjas, rojizos). Una vez más mediante una composición individual $(86,95 \%)$ y sobre todo gracias a un primer plano $(46,25 \%)$ o un plano medio $(20,26 \%)$, sobresalen los arquetipos «Preocupada por su belleza y el paso del tiempo» $(29,10 \%)$ y «Elegante y sofisticada» $(32,27 \%)$, el cual torna a «Glamurosa y chic», mujer triunfadora segura de sí misma y con la noche como escenario genuino. Continúa «Sensual y soñadora» $(25,39 \%)$ y desciende levemente «Mujer fatal» (13,22 \%); el actante masculino, específicamente en una relación de pareja, ostenta un peso reducido del 8,26\% si bien siempre hay contacto físico explícito entre mujer y hombre (beso, abrazo, roce).

Físicamente, se empieza a advertir cierta diversidad en la imagen de la mujer prescriptora de Belleza e Higiene, con algunos anuncios protagonizados por mujeres de más edad, de otra raza diferente a la caucásica habitual y de cabello corto, entre otros matices diferenciales. A su vez, su expresión se antoja más dinámica y espontánea, menos rígida y encorsetada, apoyada por un vestuario y atrezzo menos recargados y fastuosos que recalcan la estética publicitaria minimalista característica de la década.

\section{Tabla 5. Estadística descriptiva 1993-2002}

\begin{tabular}{|l|l|l|}
\hline \multicolumn{2}{|c|}{ Anuncios categoría «Belleza e Higiene» } & $\%$ \\
\hline Subcategorías & & $65,13 \%$ \\
\hline & Colonias y perfumes & $62,65 \%$ \\
\hline & Femeninos & $2,48 \%$ \\
\hline & Masculinos & $12,86 \%$ \\
\hline & Maquillaje & $17,00 \%$ \\
\hline & Tratamientos de belleza faciales & $1,65 \%$ \\
\hline & Cuidados del cuerpo & $0,88 \%$ \\
\hline & Productos cabello & $2,48 \%$ \\
\hline
\end{tabular}




\section{Tabla 5. Estadística descriptiva 1993-2002 (continuación)}

Formatos publicitarios

\begin{tabular}{|l}
\hline Lanzamiento marca / producto \\
\hline Código escritural \\
\hline
\end{tabular}

Página

Doble página

Concepto «novedad»

\section{Código escritural}

Principales elementos

Titular

$29,13 \%$

Cuerpo de texto

$27,39 \%$

Eslogan

$38,26 \%$

Simple identificación marca y producto

$28,26 \%$

Contenido predicación

Breve acotación sobre marca y producto

\section{Código tipográfico}

Recursos destacados

\section{Mayúsculas}

Signos (interrogación, exclamación)

\section{Código cromático}

Código fotográfico

\section{$\mathrm{B} / \mathrm{N}$}

Color

\section{Código fotc}

Principales planos

Primer plano

Plano medio

Plano americano

\section{Código sociocultural}

\section{Prescriptora celebrity}

Relación actantes $\mathrm{H}$ y M

Arquetipos femeninos dominantes

\begin{tabular}{|l|l|}
\hline Actrices, modelos de prestigio, diseñadoras & $17,82 \%$ \\
\hline Relación de pareja & $8,26 \%$ \\
\hline Preocupada por su belleza y el paso del tiempo & $29,10 \%$ \\
\hline Elegante y sofisticada / Glamurosa y chic & $32,27 \%$ \\
\hline Sensual y soñadora & $25,39 \%$ \\
\hline Mujer fatal & $13,22 \%$ \\
\hline Contacto físico con el producto & \\
\hline Composición individual & $9,56 \%$ \\
\hline Composición de pareja o grupal & \\
\hline
\end{tabular}

Fuente: Elaboración propia.

\section{Código gestual}

\section{Código morfológico}

\section{Composición de pareja o grupal}




\subsection{De 2003 a 2012:}

«Colonias y perfumes» (59,37 \%), en ocasiones con anuncios con versión femenina y masculina en una misma pieza, lidera una vez más el ranking de inversión publicitaria de Belleza e Higiene, ocupando «Tratamientos de belleza faciales» $(17,70 \%)$ y «Maquillaje» $(15,27 \%)$ el segundo y el tercer lugar respectivamente en la clasificación. «Productos para el cabello», «Estética corporal»y «Cuidados del cuerpo» concluyen el reparto de la categoría. A pesar de la crisis económica que azota desde la mitad de la década, es el periodo que registra una mayor inversión publicitaria. Más que al desembarco de nuevas marcas, se asiste a lanzamientos de referencias de marcas ya presentes en el mercado (46,26 \%), con una preeminencia indiscutible de enseñas internacionales. De ahí que, al margen de casos de simple identificación de marca y producto $(22,01 \%)$, se vuelvan a precisar elementos escriturales para subrayar la novedad (17,91\%), la primicia y un eslogan $(27,61 \%)$ o un escueto body copy explicativo del lanzamiento (36,94\%).

El empleo de la doble página como formato publicitario $(27,62 \%)$ crece. Mediante ella y la página $(72,38 \%)$, las promesas de «felicidad»y «éxito» resumen los anhelos de la década; «exclusividad», «lujo» y sobre todo «perfección» se esgrimen como los cauces para alcanzarlos. Estas promesas encuentran su contrapunto en modelos de mujer representados por celebridades $(25,74 \%)$ de ámbitos variados: diseño, modelaje, música y fundamentalmente cine. Como innovación, esta prescriptora relevante se identifica, con nombre y apellidos, en un 50,71\% de los anuncios, a veces incluyendo una frase entrecomillada a modo testimonial y su firma. A través de ellas o mediante prescriptoras anónimas, los arquetipos «Preocupada por su belleza y el paso del tiempo» (32,83 \%), «Glamurosa y chic» $(26,11 \%)$ y «Sensual y soñadora» $(17,91 \%)$ conviven con una «Mujer fatal» $(23,13 \%)$ que trasciende la relación de pareja hombre y mujer (8,95\% de las piezas), la cual siempre retrata una escena íntima y erótica, para connotar una mujer evocadora de lujo y poder. La «Mujer fatal» actual, dominante y rebelde, alterna con contenidos simbólicos de la mitología clásica (serpiente, manzana, ángel, ninfa, deidad...) y con reminiscencias a divas cinematográficas, desembocando en una comunicación publicitaria emocional y marcadamente aspiracional.

Esta prescriptora publicitaria, siempre esbelta y cuya diversidad se reduce prácticamente al aspecto racial, apenas mantiene relación con el producto (11,56 \%), en anuncios de estética monocromática y caracteres con remates y ornamentos, contrastando con un vestuario y atrezzo de líneas sencillas que ceden el protagonismo a la impronta de la figura femenina, mostrando mediante un plano medio (38,43\%), primer plano $(29,10 \%)$ o americano $(15,67 \%)$ una pose frecuentemente forzada que simula un «instante robado».

\section{Tabla 6. Estadística descriptiva 2003-2012}

\begin{tabular}{|l|l|c|}
\hline \multicolumn{2}{|c|}{ Anuncios categoría «Belleza e Higiene» } & $\%$ \\
\hline Subcategorías & & $59,37 \%$ \\
\hline & Colonias y perfumes & $55,21 \%$ \\
\hline & Femeninos & $4,16 \%$ \\
\hline & Masculinos & $15,27 \%$ \\
\hline
\end{tabular}




\section{Tabla 6. Estadística descriptiva 2003-2012 (continuación)}

\begin{tabular}{|c|c|c|}
\hline & Tratamientos de belleza faciales & $17,70 \%$ \\
\hline & Cuidados del cuerpo & $1,38 \%$ \\
\hline & Productos cabello & $3,12 \%$ \\
\hline & Estética corporal & $3,16 \%$ \\
\hline \multicolumn{3}{|l|}{ Formatos publicitarios } \\
\hline & Página & $72,38 \%$ \\
\hline & Doble página & $27,62 \%$ \\
\hline Lanzamiento marca / producto & Concepto «novedad» & $46,26 \%$ \\
\hline \multicolumn{3}{|l|}{ Código escritural } \\
\hline Principal elemento & Titular & $26,49 \%$ \\
\hline \multirow{4}{*}{ Contenido predicación } & Simple identificación marca y producto & $22,01 \%$ \\
\hline & Marca y producto + novedad & $17,91 \%$ \\
\hline & Marca y producto + novedad + eslogan & $27,61 \%$ \\
\hline & Breve cuerpo de texto explicativo & $36,94 \%$ \\
\hline \multicolumn{3}{|l|}{ Código tipográfico } \\
\hline Recurso destacado & Mayúsculas & $25,37 \%$ \\
\hline \multicolumn{3}{|l|}{ Código cromático } \\
\hline & $\mathrm{B} / \mathrm{N}$ & $0,74 \%$ \\
\hline & Color & $99,26 \%$ \\
\hline \multicolumn{3}{|l|}{ Código fotográfico } \\
\hline \multirow{3}{*}{ Principales planos } & Primer plano & $29,10 \%$ \\
\hline & Plano medio & $38,43 \%$ \\
\hline & Plano americano & $15,67 \%$ \\
\hline \multicolumn{3}{|l|}{ Código sociocultural } \\
\hline \multirow{2}{*}{ Prescriptora celebrity } & Actrices, modelos, diseñadoras, cantantes & $25,74 \%$ \\
\hline & Identificación prescriptora & $50,71 \%$ \\
\hline Relación actantes Hy M & Relación de pareja & $8,95 \%$ \\
\hline \multirow{4}{*}{ Arquetipos femeninos dominantes } & Preocupada por su belleza y el paso del tiempo & $32,83 \%$ \\
\hline & Glamurosa y chic & $26,11 \%$ \\
\hline & Sensual y soñadora & $17,91 \%$ \\
\hline & Mujer fatal & $23,13 \%$ \\
\hline
\end{tabular}




\section{Tabla 6. Estadística descriptiva 2003-2012 (continuación)}

Código gestual

\begin{tabular}{|l|l|l|}
\hline & Contacto físico con el producto & $11,56 \%$ \\
\hline Código morfológico & & $86,95 \%$ \\
\hline & Composición individual & $13,05 \%$ \\
\hline & Composición de pareja o grupal & 1 \\
\hline
\end{tabular}

Fuente: Elaboración propia.

\subsection{De 2013 a la actualidad:}

«Tratamientos de belleza faciales» $(17,88 \%)$ y «Maquillaje» (11,38 \%) añaden los conceptos «lifting» $\mathrm{y}$ «anti-edad» a los principios básicos de cuidado facial, constituyendo la «firmeza» y la «juventud» las promesas decisivas de este último periodo. «Colonias y perfumes» conserva su reinado $(57,71 \%)$, y la subcategoría «Estética corporal» $(8,17 \%)$, cuya inversión publicitaria se intensifica, suma nuevos servicios como depilación láser, medicina y cirugía plástica y franquicias de uñas.

Gracias a la utilización de imágenes gráficas en anuncios a una página $(69,74 \%)$ o a doble página $(30,26 \%)$, la prescriptora publicitaria de belleza, en compañía de un actante masculino $(11,76 \%)$ u otras mujeres $(7,56 \%)$ pero básica- mente en solitario $(80,67 \%)$, se manifiesta seductora, confiada y desinhibida; publicita marcas y productos, nuevos en un 53,78 \% de los casos, desde la potestad que le confiere ser famosa y admirada (31,93\%). Ante tal contundente reclamo, los elementos escriturales y tipográficos se limitan a señalar marca, producto, innovación y eslogan, recortándose a favor de los contrastes cromáticos y del dorado como símbolo de opulencia. El plano medio (51,26 \%) es el preferido para exhibir una mujer «Glamurosa y chic» $(33,61 \%)$, que supera a la clásica «Preocupada por su belleza y el paso del tiempo» $(29,41 \%)$. «Sensual y soñadora» $(19,32 \%)$ y «Mujer fatal» $(17,64 \%)$ cierran los arquetipos, gobernados siempre por una expresión de satisfacción y seguridad.

\section{Tabla 7. Estadística descriptiva 2013-2018}

\begin{tabular}{|l|l|c|}
\hline \multicolumn{4}{|c|}{ Anuncios categoría «Belleza e Higiene» } & $\%$ \\
\hline Subcategorías & & $57,71 \%$ \\
\hline & Colonias y perfumes & $55,28 \%$ \\
\hline & Femeninos & $2,43 \%$ \\
\hline & Masculinos & $11,38 \%$ \\
\hline & Maquillaje & $17,88 \%$ \\
\hline & Tratamientos de belleza faciales & $2,43 \%$ \\
\hline
\end{tabular}




\section{Tabla 7. Estadística descriptiva 2013-2018 (continuación)}

\begin{tabular}{|c|c|c|}
\hline & Productos cabello & $2,43 \%$ \\
\hline & Estética corporal & $8,17 \%$ \\
\hline \multicolumn{3}{|l|}{ Formatos publicitarios } \\
\hline & Página & $69,74 \%$ \\
\hline & Doble página & $30,26 \%$ \\
\hline Lanzamiento marca / producto & Concepto «novedad» & $53,78 \%$ \\
\hline \multicolumn{3}{|l|}{ Código escritural } \\
\hline \multirow{4}{*}{ Contenido predicación } & Simple identificación marca y producto & $23,52 \%$ \\
\hline & Marca y producto + novedad & $22,68 \%$ \\
\hline & Marca y producto + novedad + eslogan & $15,15 \%$ \\
\hline & Breve cuerpo de texto explicativo & $38,65 \%$ \\
\hline \multicolumn{3}{|l|}{ Código tipográfico } \\
\hline Recurso destacado & Mayúsculas & $23,52 \%$ \\
\hline \multicolumn{3}{|l|}{ Código cromático } \\
\hline & Color & $100,00 \%$ \\
\hline \multicolumn{3}{|l|}{ Código fotográfico } \\
\hline \multirow{3}{*}{ Principales planos } & Primer plano & $29,10 \%$ \\
\hline & Plano medio & $51,26 \%$ \\
\hline & Plano americano & $16,80 \%$ \\
\hline \multicolumn{3}{|l|}{ Código sociocultural } \\
\hline \multirow{2}{*}{ Prescriptora celebrity } & Actrices, modelos, diseñadoras, cantantes & $31,93 \%$ \\
\hline & Identificación prescriptora & $60,52 \%$ \\
\hline Relación actantes $\mathrm{H}$ y M & Relación de pareja & $11,76 \%$ \\
\hline \multirow{4}{*}{ Arquetipos femeninos dominantes } & Preocupada por su belleza y el paso del tiempo & $29,41 \%$ \\
\hline & Glamurosa y chic & $33,61 \%$ \\
\hline & Sensual y soñadora & $19,32 \%$ \\
\hline & Mujer fatal & $17,64 \%$ \\
\hline \multicolumn{3}{|l|}{ Código gestual } \\
\hline & Contacto físico con el producto & $16,80 \%$ \\
\hline \multicolumn{3}{|l|}{ Código morfológico } \\
\hline & Composición individual & $80,67 \%$ \\
\hline & Composición de pareja o grupal & $19,33 \%$ \\
\hline
\end{tabular}

Fuente: Elaboración propia. 


\section{Discusión y conclusiones}

En base a los resultados obtenidos, el análisis diacrónico de la figura de la prescriptora publicitaria de belleza en España ilustra y completa investigaciones precedentes que alegan la preponderancia de la esbeltez, la juventud y la perfección (Torres, 2007) como valores anhelados por la sociedad en general y específicamente por la consumidora de revistas de alta gama como Telva, target de marcas y productos de cuidado personal cuya publicidad es objeto del presente estudio. Si bien Almansa-Martínez y Gómez de TravesedoRojas (2017) apuntan en su análisis sobre la evolución del estereotipo de mujer en publicidad la excesiva preocupación que ésta otorga a la apariencia física en la actualidad, esta investigación revela que tal inquietud se exterioriza ya desde la década de 1960 en nuestro país, concentrándose no obstante el concepto de belleza actual en el mismo territorio que el poder y el estatus (SoleyBeltrán, 2012).

Desde entonces y hasta hoy, el recurso de la figura femenina como prescriptora publicitaria de marcas y productos de Belleza e Higiene cambia a medida que lo hace el contexto social y cultural, reflejando las modas, estéticas, estándares y tendencias imperantes; no obstante la limitación que puede conllevar la amplitud de la muestra, que comprende contextos y cosmovisiones diversos, la tarea de asesoramiento de la prescriptora publicitaria suele plasmarse invariablemente a través de dos vías históricas complementarias: el camino creativo tipificado como testimonial, hablando como usuaria del producto, agradeciendo directa o indirectamente su ayuda y remarcando las ventajas y propiedades que derivan en valores para la beneficiaria; y la fórmula de la demostración de las bondades que marca y producto conceden a la consumidora gracias a la imagen de una mujer que evidencia los resultados o los efectos de la promesa publicitada.

De este modo, se corrobora la hipótesis de partida al ser esencialmente en los patrones compositivos de los anuncios, en los arquetipos y en el grado de notoriedad de la prescriptora donde se perciben las mayores diferencias desde 1963 hasta 2018, y no tanto en los deseos y las motivaciones que subyacen a este tipo de publicidad. Como principales hallazgos a nivel compositivo, sobresalen la consolidación de página y doble página como formatos publicitarios, la sustitución del blanco y negro por el color, la depuración del código escritural y la evolución del primer plano al plano medio, entre otros. En cuanto al arquetipo femenino dominante, el modelo de mujer «Glamurosa y chic» llega a rivalizar con el tradicional de «Preocupada por su belleza y el paso del tiempo», impulsado por el incremento paulatino de celebridades como prescriptoras a las que se les vinculan tales atributos.

Los resultados relativos a publicidad de belleza certifican, en referencia a los objetivos planteados, la solidez de la comunicación emitida por una marca a través de la voz de una mujer que representa las aspiraciones del momento, y cómo ésta se convierte en un referente con el que identificarse las demás mujeres gracias a un discurso retórico de índole connotativo y de corte seductor, triunfador y cómplice. Se trata por tanto de una comunicación de mujer a mujer, donde destaca el reconocimiento y el halago por parte de otras féminas mientras que el actante masculino desempeña un papel secundario y de peso reducido, evolucionando desde un mero objeto de seducción a participar como seductor en la composición de pareja, cada vez más explícitamente erótica. A pesar de su involucración en la acción, su condición de anonimato contrasta con una prescriptora publicitaria cada vez más conocida, sien- 
do una celebridad o personalidad relevante la fuente prescriptora más codiciada por parte de marcas y productos de la categoría Belleza e Higiene, de forma que su simple presencia sea suficiente para condensar la esencia de la promesa y para transferir a la marca y al producto las cualidades de imagen que sustenta, sin olvidar su naturaleza como activo y su compromiso en relación a la estrategia de marketing a la que se supedita.

\section{Bibliografía}

Aguilera, S. (2015, 17 de diciembre). La inversión publicitaria en los meses de navidad se dispara. Marketingdirecto.com. Disponible en: https://www.marketingdirecto.com/marketing-general/publicidad/la-inversion-publicitaria-los-meses-navidad-se-dispara

Aguirre, I. (2010). El despertar del hechizo de la marca: de la seducción de la marca al poder del prescriptor. Investigación E Marketing, (106), 34-37.

Almansa-Martínez, A. \& Gómez de Travesedo-Rojas, R. (2017). El estereotipo de mujer en las revistas femeninas españolas de alta gama durante la crisis. Revista Latina de Comunicación Social, (72), 608-628.

Amos, C., Holmes, G. \& Strutton, D. (2008). Exploring the relationship between celebrity endorser effects and advertising effectiveness: A quantitative synthesis of effect size. International Journal of Advertising, 27(2), 209-234.

Aranda, A. (2018, 6 de junio). La cosmética (ya) no solo tiene que ver con la estética. Forbes. Disponible en: http:// forbes.es/business/43235/la-cosmetica-ya-no-solo-tieneque-ver-con-la-estetical

Arroyo, I. (2005). Creatividad Publicitaria y Retórica: De la metáfora a los efectos especiales. Revista ICONO14, 3(1), 155-170.

Benito, L. (2018). Una aproximación a la publicidad de cosméticos en las revistas femeninas editadas en España. grafica, 6(12), 77-84.

Cabello, F. (1999). El mercado de revistas en España. Concentración informativa. Barcelona: Ariel.

Castelló, A. \& del Pino, C. (2015). Prescriptores, marcas y tuits: el marketing de influencia. Revista Internacional de Investigación en Comunicación aDResearch ESIC, 12(12), 86-107.
Castelló, A., del Pino, C. \& Tur-Viñes, V. (2016). Estrategias de contenido con famosos en marcas dirigidas a público adolescente. Revista ICONO14, 14(1), 123-154.

Castrillo, D. \& Oksman, S. (Productores). (2007). 50 años de Spots (Documental). España: Canal de Historia y Documenta Films.

Chan, K. K. \& Misra, S. (1990). Characteristics of the opinion leader: a new dimension. Journal of Advertising, 19(3), 53-60.

Del Rosso, T. (2016). There's a cream for that: A textual analysis of beauty and body-related advertisements aimed at middle-aged women. Journal of Women \& Aging, 29(2), 185-197.

Eguizábal, R. (1998). Historia de la Publicidad. Madrid: Eresma \& Celeste.

Erdogan, B. Z. (1999). Celebrity endorsement: a literature review. Journal of Marketing Management, 15(4), 291-314.

Fowler, J. G. \& Carlson, L. (2015). The visual presentation of beauty in transnational fashion magazine advertisements. Journal of Current Issues E Research in Advertising, 36(2), 136-156.

Gallego, J. (1990). Mujeres de papel: de ¡Hola! a Vogue. La prensa femenina en la actualidad. Barcelona: Icaria.

Ganzabal, M. (2006). Nacimiento, evolución y crisis de la prensa femenina contemporánea en España. Ámbitos. Revista Internacional de Comunicación, (15), 405-420.

García, A. \& Fernández, J. M. (1990). Publicidad en TV. Los anunciantes descubren la tele. Años 1957-1967. Madrid: Cámara de Comercio e Industria.

González-Anleo, J. M., Cortés, M. \& Garcelán, D. (2018). Roles y estereotipos de género en publicidad infantil: ¿Qué ha 
cambiado en las últimas décadas? Revista Internacional de Investigación en Comunicación aDResearch ESIC, 18(18), 80-99.

Hidalgo-Marí, T. (2015). El resurgir de la mujer fatal en publicidad: La reinvención de un mito. Cuestiones de género: de la igualdad y la diferencia, (10), 394-418.

Krippendorff, K. (1989). Content analysis. In Barnouw, E., Gerbner, G., Schramm, W., Worth, T. L. \& Gross, L. (Eds.). International encyclopedia of communication (pp. 403-407). New York: Oxford University Press.

Lazarsfeld, P. F., Berelson, B. \& Gaudet, H. (1944). The people's choice: how the voter makes up his mind in a presidential campaign. Oxford: Duell, Sloan \& Pearce.

López-Aranguren, E. (2010). El análisis de contenido tradicional. En García, M., Alvira, F. e Ibáñez, J. (Comp.). El análisis de la realidad social. Métodos y técnicas de investigación (pp. 555-574). Madrid: Alianza Editorial.

Luque, S. \& Pérez, C. (2018). El empoderamiento femenino en las revistas de moda. ¿Realidad o aspiración? RIHC: Revista Internacional de Historia de la Comunicación, (10), 122-149.

Magaña, L. C. (2014). Cuestión de género: algunos aspectos clave del feminismo en la creación artística posmoderna. Cuadernos Interc.a.mbio sobre Centroamérica y el Caribe, 11(2), 319-327.

Martín, L. R. (2013). Los discursos comunicativos y sus relaciones con el consumo del siglo XXI. Revista Internacional de Investigación en Comunicación aDResearch ESIC, 7(7), 36-57.

Martínez-Sanz, R. \& González, C. (2018). Comunicación de Marca en Instagram, ¿Una Cuestión de Género? El Rol del Influencer de Moda. MCS, 7(3), 230-254.

McCracken, G. (1989). Who is the celebrity endorser? Cultural foundations of the endorsement process. Journal of Consumer Research, 16(3), 310-321.

Menéndez, M. I. \& Figueras, M. (2013). La evolución de la prensa femenina en España: de La Pensadora Gaditana a los blogs. Comunicació: Revista de Recerca i d'Anàlisi, 30(1), 25-48.

Moeran, B. (2010). The portrayal of beauty in women's fashion magazines. Fashion Theory, 14(4), 491-510.

Mondría, J. (2004). Diccionario de la comunicación comercial. Madrid: Díaz de Santos.

Ohanian, R. (1990). Construction and validation of a scale to measure celebrity endorsers' perceived expertise, trustworthiness, and attractiveness. Journal of Advertising, 19(3), $39-52$.
Real Academia Española (2018). Diccionario de la lengua española (23. ${ }^{a}$ edición).

Rey, J. (2018). La mercantilización del cuerpo masculino en la publicidad moderna. Questiones Publicitarias, 2(23), 43-56.

Roca, M. (2006). La imagen de la mujer en la prensa femenina en «Telva» (1963-2000). Comunicar, (26), 149-154.

Rumschisky, A. (2009). El valor de la utilización de personajes famosos en la comunicación publicitaria. Un análisis cuantitativo de precios para un producto de moda (Tesis Doctoral). UCM, Facultad de Ciencias de la Información.

Soley-Beltrán, P. (2012). Muñecas que hablan. Ética y estética de los modelos de belleza en publicidad y moda. Revista de Dialectología y Tradiciones Populares, 67(1), 115-146.

Taveras, J. (2014). Las estrellas de Hollywood como prescriptores en el spot publicitario: un activo para las marcas y anunciantes. Pensar la Publicidad, 8(2), 233-255.

Taveras, J. (2016). La estrella de cine como prescriptor y actante en el spot publicitario: la construcción del deseo. Comunicación: Revista Internacional de Comunicación Audiovisual, Publicidad y Estudios Culturales, (14), 80-95.

Torres, R. (2007). Revistas de moda y belleza: El contenido al servicio de la forma bella. Ámbitos. Revista Internacional de Comunicación, (16), 213-225.

Torres, E. \& García, S. (2015). Representación de la vejez en publicidad: presencia de estereotipos, prescriptores y consumidores. Opción, (2), 1083-1104.

Treviños, D. \& Díaz-Soloaga, P. (2018). Estereotipos femeninos en anuncios gráficos de marcas de lujo de moda, perfumería y cosmética. Pensar la Publicidad, 12, 145-164.

Tzoumaka, E., Tsiotsou, R. H. \& Siomkos, G. (2016). Delineating the role of endorser's perceived qualities and consumer characteristics on celebrity endorsement effectiveness. Journal of Marketing Communications, 22(3), 307-326.

Vega, S., Barredo, D. \& Merchán, A. (2019). Percepción de los comportamientos y patrones corporales asignados a la mujer en publicidad. Ámbitos. Revista Internacional de Comunicación, (44), 162-180.

Viñuelas-López, L. (2019). La publicidad de perfumes en las revistas de moda. Creatividad y Sociedad, (30), 198-220.

Wimmer, R. D. \& Dominick, J. R. (1996). Mass Media Research - an Introduction (5. ${ }^{\mathrm{a}}$ ed.). Belmont: Wadsworth Publishing Co Inc. 
